\title{
Double Inversion Recovery MR Sequence for the Detection of Subacute Subarachnoid Hemorrhage
}

J. Hodel, R. Aboukais, B. Dutouquet, E. Kalsoum, M.A. Benadjaoud, D. Chechin, M. Zins, A. Rahmouni, A. Luciani, J.-P. Pruvo, J.-P. Lejeune, and X. Leclerc

\begin{abstract}
BACKGROUND AND PURPOSE: The diagnosis of subacute subarachnoid hemorrhage is important because rebleeding may occur with subsequent life-threatening hemorrhage. Our aim was to determine the sensitivity of the 3D double inversion recovery sequence compared with CT, 2D and 3D FLAIR, 2D T2*, and 3D SWI sequences for the detection of subacute SAH.
\end{abstract}

MATERIALS AND METHODS: This prospective study included 25 patients with a CT-proved acute SAH. Brain imaging was repeated between days 14 and 16 (mean, 14.75 days) after clinical onset and included MR imaging (2D and 3D FLAIR, 2D T2*, SWI, and 3D double inversion recovery) after CT (median delay, 3 hours; range, 2-5 hours). A control group of 20 healthy volunteers was used for comparison. MR images and CT scans were analyzed independently in a randomized order by 3 blinded readers. For each subject, the presence or absence of hemorrhage was assessed in 4 subarachnoid areas (basal cisterns, Sylvian fissures, interhemispheric fissure, and convexity) and in brain ventricles. The diagnosis of subacute $\mathrm{SAH}$ was defined by the presence of at least 1 subarachnoid area with hemorrhage.

RESULTS: For the diagnosis of subacute $S A H$, the double inversion recovery sequence had a higher sensitivity compared with $C T(P<.001)$, 2D FLAIR $(P=.005), \mathrm{T}^{*}(P=.02)$, SWI, and 3D FLAIR $(P=.03)$ sequences. Hemorrhage was present for all patients in the interhemispheric fissure on double inversion recovery images, while no signal abnormality was noted in healthy volunteers. Interobserver agreement was excellent with double inversion recovery.

CONCLUSIONS: Our study showed that the double inversion recovery sequence has a higher sensitivity for the detection of subacute SAH than CT, 2D or 3D FLAIR, 2D T2*, and SWI.

ABBREVIATIONS: IVH = intraventricular hemorrhage; DIR = double inversion recovery

$\mathbf{N}$ ontraumatic subarachnoid hemorrhage accounts for 3\% of all strokes, and $85 \%$ are related to a ruptured intracranial aneurysm. ${ }^{1}$ CT is highly sensitive for the diagnosis of SAH at the acute stage. ${ }^{2}$ However, clinical symptoms may be atypical and result in delayed admission. In such patients, the diagnosis of subacute SAH is important because rebleeding may occur with subsequent life-threatening intracranial hemorrhage. ${ }^{3}$

When performed several days after symptom onset, CT does not appear reliable for the diagnosis of $\mathrm{SAH}^{4,5}$ and is outperformed by

Received June 14, 2014; accepted after revision July 11.

From the Departments of Neuroradiology (..H., B.D., E.K., J.-P.P., X.L) and Neurosurgery (R.A., J.-P.L), Hôpital Roger Salengro, Lille, France; Institut National De La Santé et De La Recherche Médicale (M.A.B.), Centre for Research in Epidemiology and Population Health, Villejuif, France; Philips Medical Systems (D.C.), Suresnes, France; Department of Radiology (I.H., M.Z.), Hôpital Saint Joseph, Paris, France; and Department of Radiology (A.R, A.L), Centre Hospitalier Universitaire, Henri Mondor, Créteil, France.

Please address correspondence to Jéro̧me Hodel, MD, Department of Neuroradiology, Hôpital Roger Salengro, Rue Emile Laine, 59037 Lille, France; e-mail: Jerome.hodel@gmail.com

Evidence-Based Medicine Level 2.

http://dx.doi.org/10.3174/ajnr.A4102 brain MR imaging in this setting. ${ }^{6}$ Indeed, FLAIR MR imaging is more sensitive than CT for SAH detection at both acute ${ }^{7-9}$ and subacute $^{10}$ stages. 3D FLAIR is even more specific than 2D FLAIR by reducing flow-related artifacts, which are known to provide falsepositive findings on 2D FLAIR. ${ }^{11}$ Nevertheless, SAH can still be misdiagnosed by using FLAIR imaging due to the time interval after onset $^{12}$ or artifacts. ${ }^{13,14} \mathrm{~T} 2^{\star}$ gradient-echo sequences are useful for subacute or chronic SAH depiction. ${ }^{6,15,16}$ Susceptibility-weighted imaging uses tissue magnetic-susceptibility differences to generate a unique contrast, based on a 3D flow-compensated gradient-echo sequence. ${ }^{17}$ Previous studies have suggested that SWI could accurately detect small amounts of SAH and intraventricular hemorrhage (IVH). ${ }^{18-20}$ A recent study focusing on the detection of microbleeds also demonstrated that SWI had a greater sensitivity for blood products than the conventional $\mathrm{T} 2{ }^{\star}$ sequence. ${ }^{21}$ However, no study available compares the diagnostic performance of $\mathrm{T} 2{ }^{\star}$ and SWI for the detection of spontaneous SAH, to our knowledge.

Double inversion recovery (DIR) MR imaging is useful for the detection of cortical lesions. ${ }^{22-24}$ This technique is based on a $3 \mathrm{D}$ 
Table 1: Demographic and clinical data at admission

\begin{tabular}{lcc}
\hline & Patients & Controls \\
\hline No. & 25 & 20 \\
Sex & $16 \mathrm{M} / 9 \mathrm{~F}$ & $13 \mathrm{M} / 7 \mathrm{~F}$ \\
Age (yr) (mean) (range) & $52(28-71)$ & 50 (26-69) \\
Fisher grading (mean) & 3 & \\
Grade 1 (No.) & 5 & \\
Grade 2 (No.) & 2 & \\
Grade 3 (No.) & 6 & \\
Grade 4 (No.) & 12 & \\
GCS (mean) (range) & $14.1(10-15)$ \\
WFNS score (mean) (range) & $1.5(1-4)$ & \\
No vascular lesion (No.) & 10 \\
Brain aneurysm (No.) & 15 & \\
Etiologic work-up (CTA and DSA) & & \\
ACA (No.) & 6 & \\
MCA (No.) & (right 2, left 2) \\
Right PcomA (No.) & 2 \\
Left vertebral artery (No.) & 1 \\
Left ICA (No.) & 1 \\
Basilar artery (No.) & 1 \\
\hline
\end{tabular}

Note:-GCS indicates Glasgow Coma Scale; WFNS, World Federation of Neurosurgical Societies; ACA, anterior communicating artery; PcomA, posterior communicating artery.

turbo spin-echo acquisition with variable refocusing flip angles (BrainView, Philips Healthcare, Best, the Netherlands; Cube, GE Healthcare, Milwaukee, Wisconsin; SPACE, Siemens, Erlangen, Germany). DIR includes 2 inversion recovery pulses designed for the suppression of both CSF and normal white matter. ${ }^{24}$ Data are still not available on the value of 3D DIR for the detection of SAH. The purpose of our study was to determine the sensitivity of the 3D DIR sequence compared with CT, 2D and 3D FLAIR, 2D T2*, and 3D SWI sequences for the detection of subacute SAH.

\section{MATERIALS AND METHODS}

\section{Patients and Brain Imaging}

This prospective monocentric study was approved by our institutional review board, and informed consent was obtained from all patients or their representatives. The inclusion criteria were as follows: 1) patient admitted for suspected SAH, 2) acute $S A H$ proved on the initial unenhanced CT scan or lumbar puncture performed at the admission, and 3) Glasgow Coma Scale score $>10$ (as required by the ethics committee). Patients with hydrocephalus or brain hematoma on the initial unenhanced CT scan were excluded.

From October to December 2013, 40 consecutive patients were diagnosed with acute and spontaneous SAH by using unenhanced CT. All CT scans were obtained at admission and were reviewed by a neuroradiologist with 20 years of experience. The diagnostic criterion for SAH on CT images was the presence of a high-attenuation area within the subarachnoid spaces. No diagnosis was based on lumbar puncture during the inclusion period. Of the 40 patients, 4 died and 11 had a Glasgow Coma Scale score of $<10$, including 4 with a brain hematoma. Finally, 25 patients fulfilled the inclusion criteria; demographic and clinical data are summarized in Table 1.

The etiologic work-up included CT angiography at admission and digital subtraction angiography (within the following 12 hours). Of the 25 patients, 15 presented with a ruptured intracranial aneurysm, which was successfully treated by coils within 48 hours after clinical onset. No patient was ventilated or received high-inspired oxygen.

From 14 to 16 days (mean, 14.75 days) after clinical onset, additional brain imaging was systematically performed, including 3T MR imaging (Achieva; Philips Healthcare) and repeated unenhanced CT (Somatom Sensation; Siemens) (median delay, 3 hours before MR imaging; range, 2-5 hours). Unenhanced spiral CT was performed from the first cervical vertebra to the vertex, with the following parameters: $120 \mathrm{kV}$; $350 \mathrm{mAs}$; collimation, $40 \times 0.6 \mathrm{~mm}$. To balance the study group, MR imaging was also performed in 20 healthy volunteers who served as controls. Healthy volunteers were scanned by using 3T MR imaging only, with the same protocol as that used in the included patients. All MR imaging examinations were performed at $3 \mathrm{~T}$ by using a $32-$ channel array head coil, without gadolinium chelate injection. MR imaging protocol included diffusion-weighted imaging, 2D and 3D FLAIR, 3D SWI, 2D T2*, and 3D DIR MR imaging. The scan duration was 20 minutes. The parameters are summarized in Table 2. The parameters of the 3D FLAIR sequence used in this study were previously optimized for CSF suppression. We tested different TR and TI values (ranging from 4000 to $8000 \mathrm{~ms}$ and from 1500 to $2800 \mathrm{~ms}$, respectively) in healthy volunteers. In this study, we also used a similar TE value for 3D DIR and 3D FLAIR sequences (near $260 \mathrm{~ms}$ ) because high effective TE values are reported to be more sensitive to increases in CSF protein concentration. ${ }^{25}$ The voxel size and acquisition time were also similar.

\section{Image Analysis}

First, MR images of healthy volunteers were randomly interspersed among the patient images. MR imaging interpretation was conducted independently by 3 blinded neuroradiologists with 25 (reader 1), 10 (reader 2), and 3 (reader 3) years of experience in neurovascular imaging.

For the detection of subacute SAH and IVH in each subject, the readers assessed each set of images (2D FLAIR, 3D FLAIR, 2D $\mathrm{T} 2{ }^{\star}$, SWI, and DIR) separately in a randomized order. The independent sessions of image interpretation were 1 week apart to avoid recall bias. The readers were blinded to clinical data (patient with SAH or healthy volunteer) and to the results of other imaging modalities. Imaging criteria for hemorrhage at the subacute stage were the following: for CT images, increased attenuation values compared with the normal CSF; for DIR and FLAIR images, increased signal intensity compared with the normal CSF; and for $\mathrm{T} 2{ }^{*}$ and susceptibility-weighted images, decreased signal intensity compared with the normal CSF. Multiplanar analysis was available for CT, 3D FLAIR, 3D SWI, and 3D DIR MR images, including axial, coronal, and sagittal reformations. The 3D susceptibility-weighted images were available in both average mode and minimum intensity projection.

For each subject and each set of images, the readers were asked to assess visually the presence or absence of hemorrhage according to a 2-point scale ( 0 , no signal abnormality; 1 , signal abnormality) within 4 subarachnoid areas (basal cisterns, Sylvian fissures, interhemispheric fissure, and brain convexity) and 1 ventricular area (including the fourth, the third, or the lateral ventricles). The basal cisterns included the perimesencephalic and prepontine cisterns and the cisterna magna. 


\begin{tabular}{|c|c|c|c|c|c|}
\hline & 2D FLAIR & 2D T2* & 3D FLAIR & 3D SWI & 3D DIR \\
\hline Acquisition plane & Axial & Axial & Sagittal & Sagittal & Sagittal \\
\hline TR/TE (ms) & $11,000 / 125$ & $1077 / 16$ & $4800 / 267$ & $13 / 19$ & $5500 / 255$ \\
\hline $\mathrm{TI}$ (ms) & 2800 & - & 1650 & - & $2600 / 625$ \\
\hline Acquired voxel size $(\mathrm{mm})$ & $0.8 \times 1.4 \times 4$ & $0.9 \times 1.2 \times 4$ & $1.2 \times 1.2 \times 1.2$ & $1.2 \times 1.2 \times 1.2$ & $1.2 \times 1.2 \times 1.2$ \\
\hline Bandwidth (Hz) & 271 & 216 & 1433 & 172 & 1433 \\
\hline Echo-train length & 31 & - & 182 & - & 173 \\
\hline No. of sections & 36 & 36 & 280 & 280 & 280 \\
\hline SENSE & 1.4 & - & 2.5 & 2.5 & 2.5 \\
\hline CLEAR & Yes & Yes & Yes & Yes & Yes \\
\hline Fat suppression & No & No & SPIR & No & SPIR \\
\hline Acquisition time & $3 \mathrm{~min}$ & $3 \mathrm{~min}$ & $3 \mathrm{~min} 20 \mathrm{sec}$ & $3 \mathrm{~min}$ & $4 \mathrm{~min}$ \\
\hline
\end{tabular}

Note:-SENSE indicates sensitivity encoding; CLEAR, inhomogeneity correction; SPIR, spectral presaturation with inversion recovery.

Table 3: Interobserver agreement among readers 1,2 , and 3 for each set of images according to the area considered

\begin{tabular}{|c|c|c|c|c|c|c|}
\hline & \multirow[b]{2}{*}{ CT } & \multicolumn{5}{|c|}{ MRI } \\
\hline & & 2D FLAIR & 3D FLAIR & 2D T2* & 3D SWI & 3D DIR \\
\hline \multicolumn{7}{|l|}{ Total } \\
\hline R1 vs R2 (95\% Cl) & $1(1-1)$ & $0.85(0.77-0.93)$ & $0.79(0.69-0.89)$ & $0.84(0.76-0.91)$ & $0.79(0.72-0.87)$ & $0.94(0.91-0.97)$ \\
\hline Rl vs R3 (95\% Cl) & 0.94 (0.85-1) & $0.68(0.57-0.79)$ & $0.65(0.53-0.76)$ & $0.74(0.65-0.83)$ & $0.64(0.55-0.73)$ & $0.90(0.87-0.93)$ \\
\hline R2 vs R3 (95\% Cl) & $0.94(0.85-1.02)$ & $0.70(0.60-0.81)$ & $0.70(0.59-0.80)$ & $0.72(0.63-0.81)$ & $0.73(0.65-0.81)$ & $0.93(0.91-0.96)$ \\
\hline \multicolumn{7}{|l|}{ Subarachnoid } \\
\hline \multicolumn{7}{|l|}{ Interhemispheric } \\
\hline R1 vs R2 (95\% Cl) & $1(1-1)$ & $0.91(0.74-1)$ & $0.83(0.60-1)$ & $0.76(0.54-0.98)$ & $0.68(0.44-0.92)$ & $1(1-1)$ \\
\hline R1 vs R3 (95\% CI) & $1(1-1)$ & $0.83(0.60-1.06)$ & $0.76(0.50-1.02)$ & $0.87(0.70-1.05)$ & $0.44(0.11-0.77)$ & $1(1-1)$ \\
\hline R2 vs R3 $(95 \% \mathrm{Cl})$ & $1(1-1)$ & $0.91(0.74-1.08)$ & $0.76(0.50-1.02)$ & $0.77(0.56-0.99)$ & $0.63(0.39-0.88)$ & $1(1-1)$ \\
\hline \multicolumn{7}{|l|}{ Sylvian fissures } \\
\hline R1 vs R2 (95\% Cl) & $1(1-1)$ & $1(1-1)$ & $1(1-1)$ & $0.94(0.83-1)$ & $0.82(0.63-1)$ & 0.95 (0.88-1) \\
\hline R1 vs R3 (95\% Cl) & $1(1-1)$ & $0.88(0.66-1.11)$ & $1(1-1)$ & $1(1-1)$ & $0.59(0.31-0.86)$ & $0.90(0.81-1)$ \\
\hline R2 vs R3 (95\% Cl) & $1(1-1)$ & 0.88 (0.66-1.11) & $1(1-1)$ & $0.94(0.83-1.06)$ & $0.73(0.50-0.96)$ & 0.95 (0.89-1.02) \\
\hline \multicolumn{7}{|l|}{ Convexity } \\
\hline R1 vs R2 (95\% Cl) & $1(1-1)$ & $0.83(0.68-0.98)$ & $0.56(0.27-0.84)$ & $0.73(0.57-0.89)$ & $0.63(0.43-0.84)$ & $0.90(0.85-0.95)$ \\
\hline R1 vs R3 (95\% Cl) & $0.83(0.60-1.07)$ & $0.61(0.42-0.81)$ & $0.48(0.23-0.73)$ & $0.60(0.42-0.78)$ & $0.63(0.44-0.83)$ & $0.87(0.81-0.92)$ \\
\hline R2 vs R3 (95\% Cl) & $0.83(0.60-1.07)$ & $0.60(0.40-0.79)$ & $0.56(0.33-0.80)$ & $0.56(0.37-0.74)$ & $0.70(0.55-0.86)$ & $0.90(0.85-0.95)$ \\
\hline \multicolumn{7}{|l|}{ Basal cisterns } \\
\hline R1 vs R2 (95\% Cl) & $1(1-1)$ & $0.33(-0.33-0.98)$ & $0.70(0.41-0.99)$ & 0.74 (0.39-1) & $0.64(0.36-0.92)$ & $0.94(0.88-1)$ \\
\hline R1 vs R3 (95\% CI) & $1(1-1)$ & $0.43(-0.07-0.92)$ & $0.25(-0.20-0.70)$ & $0.45(-0.01-0.98)$ & $0.25(-0.13-0.62)$ & $0.89(0.82-0.97)$ \\
\hline R2 vs R3 $(95 \% \mathrm{Cl})$ & $1(1-1)$ & $0.39(-0.29-1.07)$ & $0.30(-0.17-0.77)$ & $0.74(0.39-1.10)$ & $0.41(0.09-0.73)$ & $0.95(0.90-1)$ \\
\hline \multicolumn{7}{|l|}{ Intraventricular } \\
\hline R1 vs R2 (95\% Cl) & $1(1-1)$ & $1(1-1)$ & $1(1-1)$ & $1(1-1)$ & $0.95(0.85-1)$ & $1(1-1)$ \\
\hline R1 vs R3 $(95 \% \mathrm{CI})$ & $1(1-1)$ & $0(-1.12-1.12)$ & $0(-1.95-1.95)$ & $0.66(0.19-1.13)$ & $0.86(0.70-1.02)$ & $0(-1.38-1.38)$ \\
\hline R2 vs R3 (95\% Cl) & $1(1-1)$ & $0(-1.12-1.12)$ & $0(--1.95-1.95)$ & $0.66(0.19-1.13)$ & $0.82(0.64-1)$ & $0(-1.38-1.38)$ \\
\hline
\end{tabular}

Note:-R1-R3 indicate readers 1-3; Total, all the subarachnoid and ventricular areas; Convexity, bilateral frontal, parietal, temporal, and occipital convexity areas; Basal cisterns, perimesencephalic and prepontine cisterns and cisterna magna.

\section{Statistical Analysis}

Statistical analyses were performed by using SAS software, Version 9.3 (SAS Institute, Cary, North Carolina). First, we evaluated the level of interobserver agreement for the detection of signal abnormalities by using CT and MR imaging (Cohen $\kappa$ test). $\kappa$ values between 0.4 and 0.6 suggested moderate agreement; $\kappa$ values between 0.6 and 0.8 , good agreement; and values higher than 0.8 , excellent agreement. Comparison of $\kappa$ values was performed. Second, disagreements between readers were resolved in consensus. Third, we compared the number of patients diagnosed with at least 1 subarachnoid and/or ventricular area with subacute hemorrhage (diagnosis of SAH and IVH, respectively) among CT, 2D FLAIR, 3D FLAIR, T2* SWI, and DIR MR images by using the McNemar $\chi^{2}$ test.

\section{RESULTS}

MR images were assessable for all subjects, with no motion artifacts.

\section{Interobserver Agreement}

Detailed results are shown in Table 3. Interobserver agreement was excellent with DIR for all the subarachnoid regions considered. Agreement between readers 1 and 2 was significantly better with DIR than with 2D FLAIR $(P=.03)$, 3D FLAIR $(P=.002)$, T2* $(P<.001)$, and SWI $(P<.001)$. Agreement between readers 1 and 3 and between readers 2 and 3 was significantly better with DIR than with the other MR images $(P<.001)$. Agreement between readers 1 and 2 was significantly improved by the use of $2 \mathrm{D}$ FLAIR compared with 3D FLAIR for the detection of SAH at the brain convexity $(P=.02)$. Agreement between readers 1 and 3 was significantly improved by the use of 3D FLAIR compared with $2 \mathrm{D}$ FLAIR for the detection of SAH at the basal cisterns $(P<.001)$.

\section{Detection of Subacute SAH}

Consensus results are summarized in Table 4. In healthy volunteers, there was no signal abnormality detected by any of the readers by using DIR or other MR imaging. 
Table 4: Number of patients with at least 1 subarachnoid and/or ventricular signal abnormality (diagnosis of SAH and IVH, respectively) for each imaging modality after consensus among readers

\begin{tabular}{lcccccc}
\hline & & \multicolumn{5}{c}{ MRI } \\
\cline { 2 - 7 } & & 2D & 3D & 2D & 3D & 3D \\
& CT & FLAIR & FLAIR & T2* $^{*}$ & SWI & DIR \\
\hline Patients with SAH & 7 & 12 & 15 & 14 & 15 & 25 \\
Interhemispheric $^{\mathrm{a}}$ & 0 & 6 & 7 & 13 & 15 & 25 \\
Sylvian fissures $^{\mathrm{a}}$ & 4 & 4 & 4 & 9 & 8 & 17 \\
Convexity $^{\mathrm{a}}$ & 3 & 8 & 8 & 10 & 11 & 19 \\
Basal cisterns $^{\mathrm{a}}$ & 0 & 1 & 5 & 4 & 7 & 23 \\
Patients with IVH & 0 & 0 & 0 & 3 & 5 & 0 \\
\hline
\end{tabular}

${ }^{a}$ Number of patients with at least 1 subarachnoid signal abnormality.

With DIR images, all patients presented with at least 2 subarachnoid areas of SAH. Conversely, there were no subarachnoid signal abnormalities in 15 patients on CT, 13 on 2D FLAIR, 11 on $\mathrm{T}^{*}$, and 10 on 3D FLAIR and SWI. Indeed, for the diagnosis of SAH (ie, a patient with at least 1 area of subarachnoid signal abnormality), DIR was more sensitive than CT $(P<.001)$, 2D FLAIR $(P=.005), 2 \mathrm{D} \mathrm{T} 2^{\star}(P=.02)$, SWI $(P=.03)$, and 3D FLAIR $(P=.03)$. Regarding the diagnosis of SAH, there was no significant difference among T2*, SWI, and 2D and 3D FLAIR.

For the diagnosis of $\mathrm{SAH}$, there were 17 disagreements among readers (ie, SAH detected by 1 reader but ruled out by the other 2 readers), including 2 disagreements with the T2* sequence, 5 with 2D FLAIR, 3 with 3D FLAIR, and 7 with 3D SWI. Reasons for disagreement included the following: hypointensity judged after consensus to be too faint or related to a vessel on $\mathrm{T} 2{ }^{*}$ or SWI; hyperintensity that could be related to CSF flow-related artifacts on 2D FLAIR; and hyperintensity within the brain sulci judged too subtle to diagnose SAH on 3D FLAIR. There was no disagreement with the 3D DIR sequence.

\section{Detection of Subacute IVH}

On consensus analysis, T2* and SWI detected 3 and 5 patients, respectively, with a small amount of bleeding in both occipital horns of the lateral ventricles, while CT, 2D FLAIR, 3D FLAIR, and DIR did not. SWI was more sensitive than CT and all other MR imaging for the diagnosis of $\operatorname{IVH}(P=.03)$. However, T2* did not prove to be significantly better than $2 \mathrm{D}$ and 3D FLAIR $(P=$ .08 ). The 5 patients with subacute IVH detected on SWI (4 men/1 woman; mean age, 52.6 years; range, $31-71$ years) also had at least 2 subarachnoid areas with SAH on DIR images.

For the diagnosis of IVH (ie, at least 1 area with IVH), there were 7 disagreements among the readers: 1 each with $\mathrm{T} 2{ }^{\star}, 2 \mathrm{D}$ FLAIR, and 3D FLAIR MR images, and 2 each with 3D DIR and 3D SWI. Reasons for disagreement included hyperintensity on DIR and FLAIR images that could be related to CSF transependymal resorption.

Figure 1 shows the absence of artifacts or subarachnoid hyperintensities on DIR images in a healthy volunteer. Figures 2-4 illustrate the improved sensitivity of the 3D DIR sequence, in comparison with other MR images, for the detection of subacute SAH.

\section{DISCUSSION}

Our study showed that the DIR sequence had a higher sensitivity for the detection of subacute SAH than CT and conventional

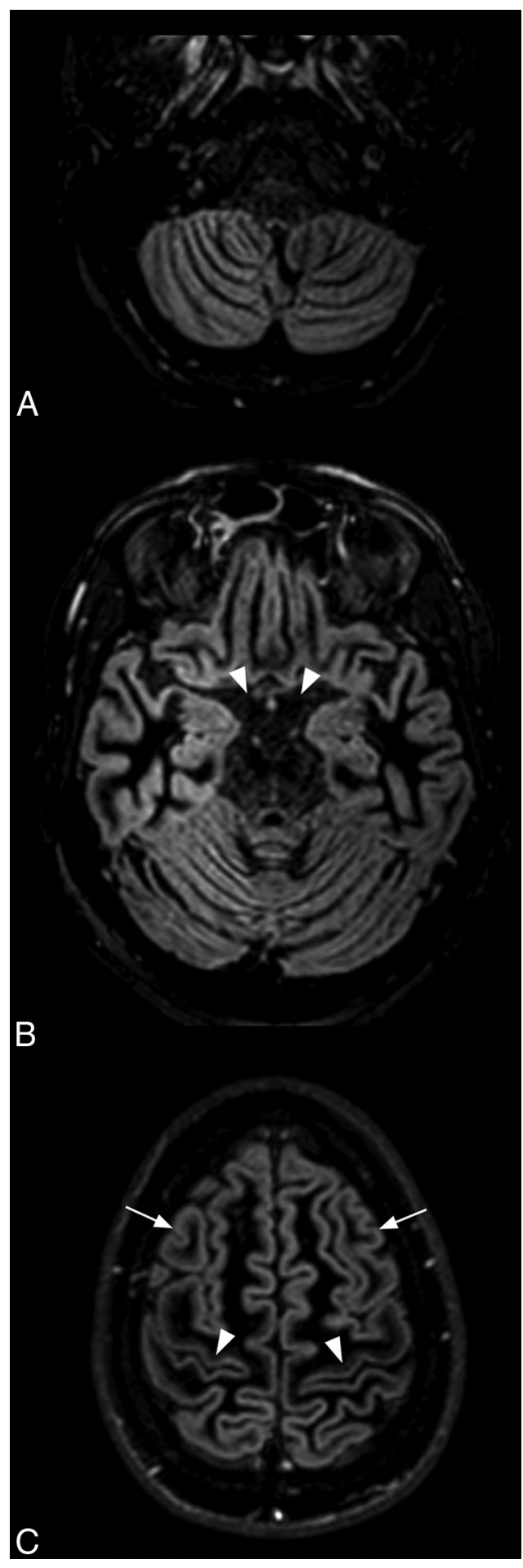

FIG 1. Axial DIR images in a 37-year-old healthy volunteer showing the basal cisterns $(A$ and $B)$ and the subarachnoid spaces at the brain convexity $(C)$. In all the healthy volunteers scanned with the DIR sequence, the CSF appeared hypointense without CSF flow-related artifacts ( $B$, arrowheads). Note the regional variation of gray matter signal intensity by using DIR, such as the difference between the prefrontal (C, arrows) and motor (C, arrowheads) cortices. The absence of CSF signal abnormality on DIR images in all the healthy volunteers and the strong agreement among readers suggest that the hyperintensities observed in patients with SAH by using DIR were not linked to artifacts. 


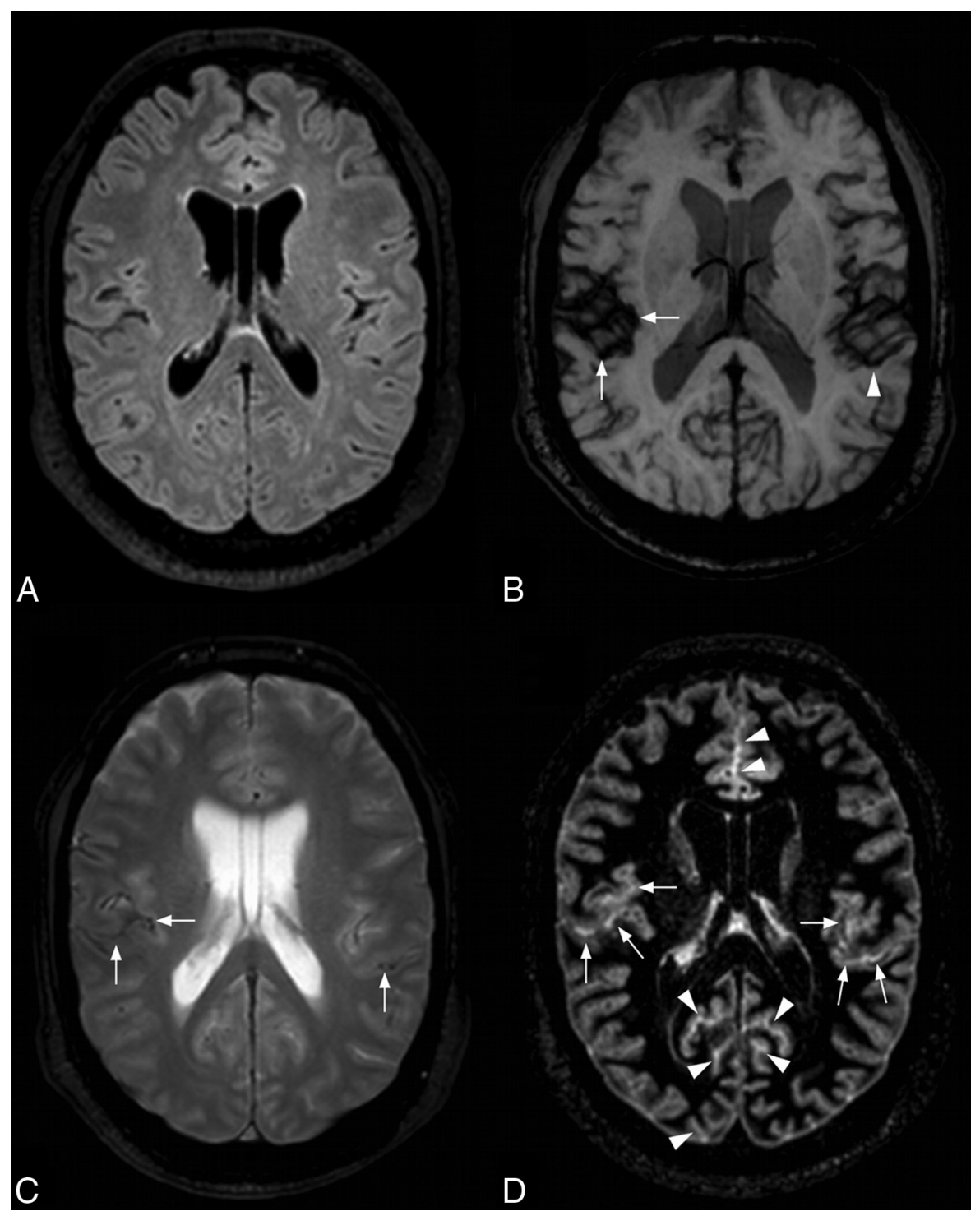

FIG 2. Subacute SAH related to an aneurysm of the anterior communicating artery in a 42-year-old woman. No signal abnormality is visible on 3D FLAIR (A). SAH is visible by using axial SWI minimum-intensity-projection reformations in the right Sylvian fissure ( $B$, arrows). The hypointensity visible in the left Sylvian fissure on SWI was considered a cortical vein by the 3 blinded readers by using both average and minimumintensity-projection reformations ( $B$, arrowhead). On the axial T2* image, SAH is bilateral, involving the Sylvian fissures (C, arrows). The DIR sequence reveals extensive SAH prevailing in the Sylvian fissures ( $D$, arrows) and interhemispheric and occipital sulci ( $D$, arrowheads).

brain MR imaging, including 2D FLAIR, 3D FLAIR, 2D T2* ${ }^{*}$ and 3D SWI. DIR appeared reliable for all the subarachnoid regions, while the sensitivity of the other imaging modalities was dependent on the anatomic distribution of hemorrhage.

In patients with subacute SAH, CT revealed subarachnoid high-attenuation abnormalities in only 7 of the 25 patients. This finding is in agreement with previous studies demonstrating the rapid attenuation changes according to the decreased concentration in hemoglobin in the time course of $\mathrm{SAH}^{4,5}$ In our institution, CT is systematically performed 2 weeks after the onset to rule out potential hydrocephalus or rebleeding.

The MR imaging appearance of SAH differs from that of cerebral hematoma because the SAH signal results from blood combined with CSF. ${ }^{8}$ Detection of SAH is related to the difference of $\mathrm{T} 1$ and $\mathrm{T} 2$ relaxation times among blood, normal CSF, and brain parenchyma. FLAIR is the reference method for the diagnosis of
SAH at the subacute stage due to CSF suppression, which improves the image contrast. On FLAIR images, the subarachnoid signal intensity is directly related to cellularity and protein levels. ${ }^{14}$ The T1-shortening effects of higher protein concentrations in bloody CSF explain why SAH can be identified by using FLAIR. $^{8,26}$ However, SAH can still be misdiagnosed by using FLAIR imaging due to the time interval between symptom onset and brain imaging ${ }^{12}$ or image artifacts. ${ }^{13,14}$

$\mathrm{T}^{*}$ is considered useful for the diagnosis of subacute or chronic SAH. ${ }^{6,16}$ This sequence appears effective in detecting a prior SAH indicating the location of a ruptured aneurysm. ${ }^{16}$ On $\mathrm{T} 2{ }^{*}$ images, SAH signal is dependent on the state of oxygenation and the integrity of red blood cell walls. A limitation of the $\mathrm{T} 2{ }^{*}$ sequence is detection of SAH, particularly at the skull base. Most interesting in the present study, SWI was not significantly better than conventional $\mathrm{T}^{*}$ for the diagnosis of $\mathrm{SAH}$. Indeed, as pre- 


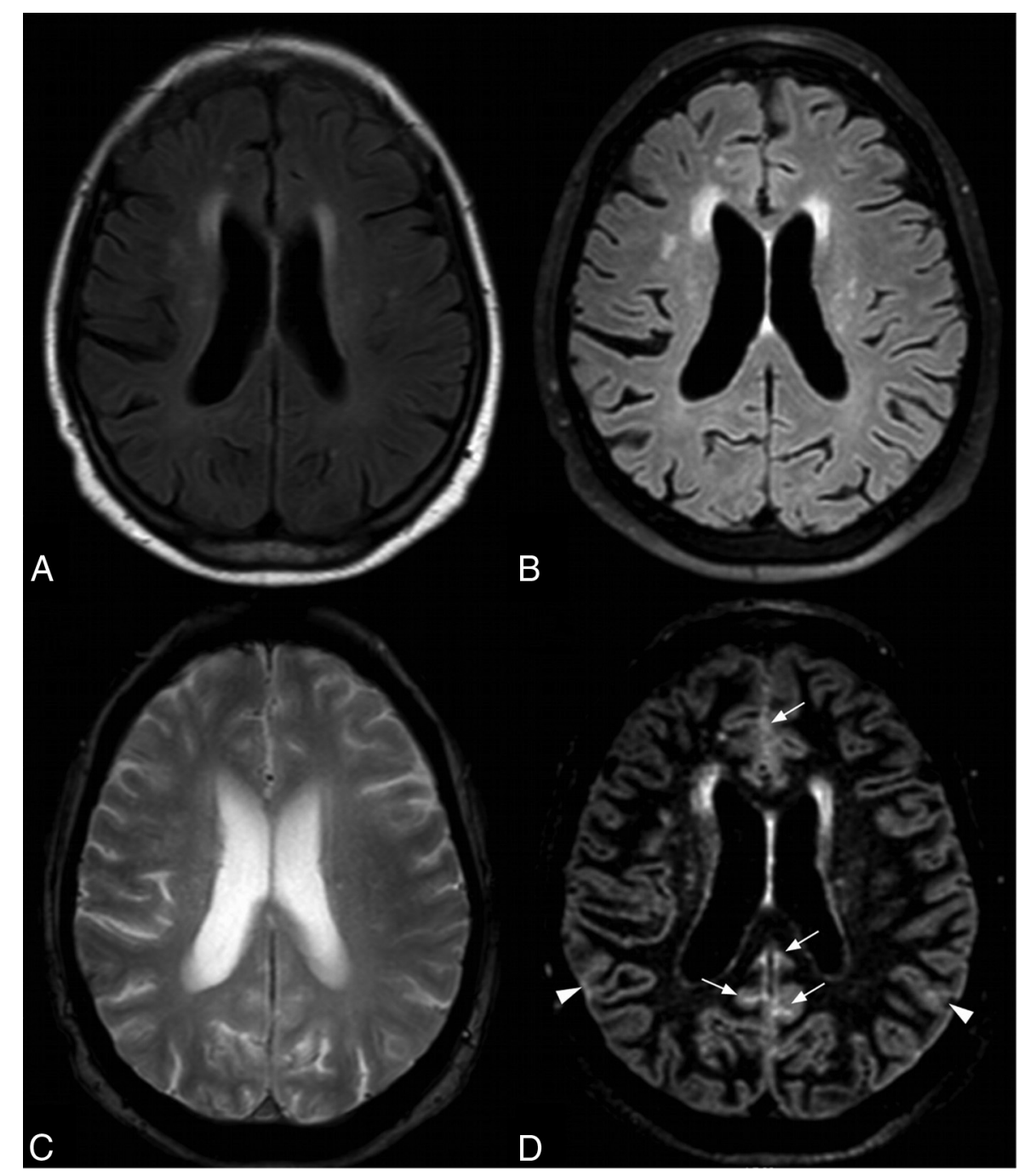

FIG 3. Subacute SAH related to an aneurysm of the anterior communicating artery in a 63-year-old man. With 2D FLAIR (A), 3D FLAIR (B), or T2* (C) images, no subacute hemorrhage is visible. Conversely, marked subarachnoid signal abnormalities along the anterior and posterior interhemispheric sulci are observed by using DIR ( $D$, arrows). SAH involving the parietal lobes is also visible ( $D$, arrowheads).

viously suggested, ${ }^{20}$ hypointense cortical veins may be difficult to distinguish from SAH on both average and minimum-intensityprojection SWI. In addition, by using SWI, the signal of SAH may be similar to the low signal of CSF. This similarity may explain why the interobserver agreement was significantly lower by using T2* and SWI compared with DIR. However, in agreement with previous studies, ${ }^{19,20} \mathrm{~T} 2^{*}$ and SWI were particularly useful for detecting IVH, while FLAIR and DIR sequences failed to detect small amounts of intraventricular bleeding. Such findings may suggest that both SWI and DIR should be performed in patients with suspected SAH.

The detection of SAH may be improved in 3 ways: 1) by reducing vascular and CSF flow-related artifacts, 2) by enhancing SAH-to-background contrast, and 3) by improving spatial resolution. There are several potential advantages of using $3 \mathrm{D}$ sequences with thinner sections such as DIR for the detection of SAH. First, the decrease in section thickness may improve SAH detection by reducing the partial volume effects. Second, while 2D sequences are prone to flow-related artifacts, ${ }^{27} 3 \mathrm{D}$ acquisition mode dramatically reduces them by using thicker volume. ${ }^{28}$ In- deed, subarachnoid signal abnormalities observed on DIR images cannot be related to slow arterial blood flow; this feature further improves the specificity of this technique for the diagnosis of SAH. Third, 3D sequences, with their higher SNR and isotropic voxel size, allow image postprocessing with reformations in arbitrary planes, which, in turn, may improve the conspicuity of signal abnormalities.

In our study, 3D DIR identified significantly more patients with SAH than 3D FLAIR and 3D SWI performed with the same voxel size, suggesting that DIR is inherently more sensitive for detecting subacute SAH. As with FLAIR, DIR suppresses the CSF signal, improving the depiction of local T1-shortening related to $\mathrm{SAH}$. The additional inversion recovery pulse, designed for white matter suppression, may further improve the contrast between $\mathrm{SAH}$ and brain tissue. The increased signal intensity of SAH observed with DIR may also be related to a different dynamic range of the signal that automatically displays optimized predefined values for window width and window level, with subsequent maximization of contrast among tissues of interest. Indeed, the combination of 2 inversion recovery pulses with DIR appears 


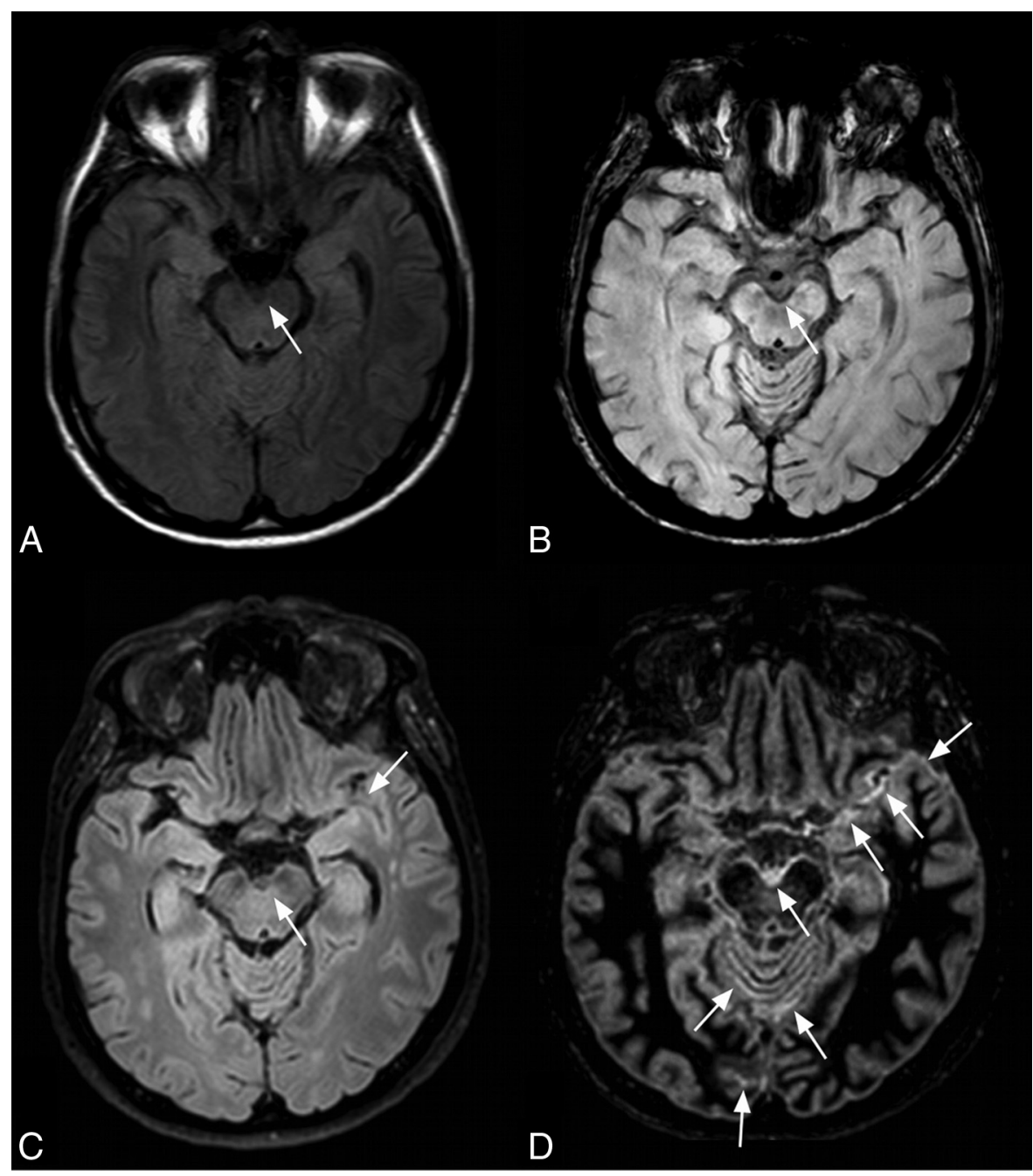

FIG 4. Subacute SAH related to an aneurysm of the left internal carotid artery in a 25-year-old woman. With 2D FLAIR images, the detection of SAH is challenging due to potential CSF flow-related artifacts $(A$, arrow). With an axial average $S W I$ reformat, a slight rim of hemorrhage is visible within the interpeduncular fossa ( $B$, arrow). On the 3D FLAIR image, SAH is subtle due to a lack of contrast (C, arrows). With DIR images, SAH is obvious, with a marked hyperintensity within the interpeduncular fossa, vermis, and left Sylvian fissure (D, arrows). Note the marked signal intensity of SAH with DIR compared with the other MR images.

particularly suitable for detecting SAH. Furthermore, DIR is also less prone to susceptibility artifacts than $\mathrm{T} 2{ }^{\star}$ or SWI because of the TSE readout used, explaining its reliability for detecting infraand supratentorial SAH. Indeed, the excellent interobserver agreement with DIR may be related to an increased signal of SAH combined with reduced artifacts.

Our study has several limitations. First, the diagnosis of SAH relied on CT at admission, but there was no standard of reference to determine the exact distribution of SAH at the subacute stage. Therefore, we only analyzed the sensitivity for detecting subacute $\mathrm{SAH}$, instead of standard accuracy parameters. The drastic improvement in SAH detection with DIR may raise doubts about the specificity of the subarachnoid signal abnormalities observed in patients. All the areas with SAH visible on FLAIR, T2*, and SWI were also detected with DIR. In addition, the absence of CSF signal abnormality on DIR images for all the healthy volunteers and the excellent agreement among readers strongly suggest that the DIR hyperintensities observed in patients were specific to SAH and thus not related to artifacts. Because the DIR sequence allows a clear delineation between the subarachnoid space and the cor- tex, a cortical lesion would have been easily distinguished from $\mathrm{SAH}$. In vitro or experimental studies could be required to confirm these findings. Second, due to the study design, only patients with Glasgow Coma Scale scores $>10$ at the time of admission were included; this choice explained the relatively small number of patients with subacute SAH. This drawback also explains the small amount of SAH (Fisher 1 or 2) detected on CT performed at admission in 7 of the 25 patients. However, such limited bleeding may further highlight the improved sensitivity of the 3D DIR sequence. Third, we did not evaluate the sensitivity of DIR at acute or more delayed stages. The marked SAH hyperintensity observed on DIR images may suggest that this sequence could also be useful at the chronic stage. Because 15 of the 25 patients underwent endovascular treatment, coils may have led to susceptibility artifacts, particularly when using T2* and SWI, which should temper the claim of a fully blinded assessment.

Differences in voxel size and the availability of multiplanar reformations were found among the $2 \mathrm{D}$ and 3D MR images. Indeed, each sequence was optimized before the study to improve the detection of SAH. Because we compared DIR with 2 other 3D 
sequences with the same voxel size (3D FLAIR and 3D SWI), our results suggest that DIR may be inherently more sensitive for detecting subacute SAH. It would have been possible to reformat the 3D sequences (FLAIR, SWI, and DIR) into axial sections that matched the section thickness and acquisition plane of the $2 \mathrm{D}$ images (FLAIR and T2*). However, such an approach would have decreased the image quality and would not have fully used the ability of 3D sequences to acquire thin sections with an optimal signal-to-noise ratio.

\section{CONCLUSIONS}

Overall, the results of this study suggest that the DIR sequence provides a greater sensitivity for the detection of SAH than CT, 2D or 3D FLAIR, 2D T2*, and 3D SWI. DIR may be a promising tool for the diagnosis of SAH in patients admitted several days after the onset of symptoms.

Disclosures: David Chechin-UNRELATED: Employment: Philips Healthcare, Comments: As an employee of Philips Healthcare, I have supported this work. Alain Luciani-UNRELATED: Payment for Development of Educational Presentations: GE Healthcare, Comments: teaching on multienergy CT. Jean-Paul Lejeune-UNRELATED: Payment for Lectures (including service on Speakers Bureaus): BrainLab, Comments: lecture during the Congress of the Société de Neurochirurgie de Langue Française; Travel/Accommodations/Meeting Expenses Unrelated to Activities Listed: B. Braun, Comments: Congress of the Société Française de Neurochirurgie (accommodation expenses).

\section{REFERENCES}

1. van Gijn J, Rinkel GJ. Subarachnoid haemorrhage: diagnosis, causes and management. Brain 2001;124:249-78

2. Backes D, Rinkel GJ, Kemperman H, et al. Time-dependent test characteristics of head computed tomography in patients suspected of nontraumatic subarachnoid hemorrhage. Stroke 2012;43:2115-19

3. Connolly ES Jr, Rabinstein AA, Carhuapoma JR, et al. Guidelines for the management of aneurysmal subarachnoid hemorrhage: a guideline for healthcare professionals from the American Heart Association/American Stroke Association. Stroke 2012;43:1711-37

4. Fainardi E, Chieregato A, Antonelli V, et al. Time course of CT evolution in traumatic subarachnoid haemorrhage: a study of 141 patients. Acta Neurochir (Wien) 2004;146:257-63, discussion 263

5. van Gijn J, van Dongen KJ. The time course of aneurysmal haemorrhage on computed tomograms. Neuroradiology 1982;23:153-56

6. Mitchell P, Wilkinson ID, Hoggard N, et al. Detection of subarachnoid haemorrhage with magnetic resonance imaging. J Neurol Neurosurg Psychiatry 2001;70:205-11

7. Noguchi K, Ogawa T, Inugami A, et al. Acute subarachnoid hemorrhage: MR imaging with fluid-attenuated inversion recovery pulse sequences. Radiology 1995;196:773-77

8. Noguchi K, Seto H, Kamisaki Y, et al. Comparison of fluid-attenuated inversion-recovery MR imaging with CT in a simulated model of acute subarachnoid hemorrhage. AJNR Am J Neuroradiol 2000;21:923-27

9. Woodcock RJ Jr, Short J, Do HM, et al. Imaging of acute subarachnoid hemorrhage with a fluid-attenuated inversion recovery sequence in an animal model: comparison with non-contrast-enhanced CT. AJNR Am J Neuroradiol 2001;22:1698-703

10. Noguchi K, Ogawa T, Seto H, et al. Subacute and chronic subarach- noid hemorrhage: diagnosis with fluid-attenuated inversion-recovery MR imaging. Radiology 1997;203:257-62

11. Lummel $\mathrm{N}$, Schoepf $\mathrm{V}$, Burke $\mathrm{M}$, et al. 3D fluid-attenuated inversion recovery imaging: reduced CSF artifacts and enhanced sensitivity and specificity for subarachnoid hemorrhage. AJNR Am J Neuroradiol 2011;32:2054-60

12. Mohamed M, Heasly DC, Yagmurlu B, et al. Fluid-attenuated inversion recovery MR imaging and subarachnoid hemorrhage: not a panacea. AJNR Am J Neuroradiol 2004;25:545-50

13. Stuckey SL, Goh TD, Heffernan T, et al. Hyperintensity in the subarachnoid space on FLAIR MRI. AJR Am J Roentgenol 2007; 189:913-21

14. Maeda M, Yagishita A, Yamamoto T, et al. Abnormal hyperintensity within the subarachnoid space evaluated by fluid-attenuated inversion-recovery MR imaging: a spectrum of central nervous system diseases. Eur Radiol 2003;13(suppl 4):L192-201

15. Inoue $\mathrm{T}$, Takada S, Shimizu H, et al. Signal changes on $\mathrm{T} 2^{\star}$-weighted magnetic resonance imaging from the acute to chronic phases in patients with subarachnoid hemorrhage. Cerebrovasc Dis 2013;36: 421-29

16. Imaizumi T, Chiba M, Honma T, et al. Detection of hemosiderin deposition by $\mathrm{T}^{\star}{ }^{*}$-weighted MRI after subarachnoid hemorrhage. Stroke 2003;34:1693-98

17. Haacke EM, Xu Y, Cheng YC, et al. Susceptibility weighted imaging (SWI). Magn Reson Med 2004;52:612-18

18. Mittal S, Wu Z, Neelavalli J, et al. Susceptibility-weighted imaging: technical aspects and clinical applications, part 2. AJNR Am J Neuroradiol 2009;30:232-52

19. Verma RK, Kottke R, Andereggen L, et al. Detecting subarachnoid hemorrhage: comparison of combined FLAIR/SWI versus CT. Eur J Radiol 2013;82:1539-45

20. Wu Z, Li S, Lei J, et al. Evaluation of traumatic subarachnoid hemorrhage using susceptibility-weighted imaging. AJNR Am J Neuroradiol 2010;31:1302-10

21. Cheng AL, Batool S, McCreary CR, et al. Susceptibility-weighted imaging is more reliable than $\mathrm{T}^{*}$-weighted gradient-recalled echo MRI for detecting microbleeds. Stroke 2013;44:2782-86

22. Geurts JJ, Pouwels PJ, Uitdehaag BM, et al. Intracortical lesions in multiple sclerosis: improved detection with 3D double inversionrecovery MR imaging. Radiology 2005;236:254-60

23. Nelson F, Poonawalla AH, Hou P, et al. Improved identification of intracortical lesions in multiple sclerosis with phase-sensitive inversion recovery in combination with fast double inversion recovery MR imaging. AJNR Am J Neuroradiol 2007;28:1645-49

24. Pouwels PJ, Kuijer JP, Mugler JP 3rd, et al. Human gray matter: feasibility of single-slab 3D double inversion-recovery high-spatial-resolution MR imaging. Radiology 2006;241:873-79

25. Melhem ER, Jara H, Eustace S. Fluid-attenuated inversion recovery MR imaging: identification of protein concentration thresholds for CSF hyperintensity. AJR Am J Roentgenol 1997;169:859-62

26. Bakshi R, Kamran S, Kinkel PR, et al. Fluid-attenuated inversionrecovery $M R$ imaging in acute and subacute cerebral intraventricular hemorrhage. AJNR Am J Neuroradiol 1999;20:629-36

27. Bakshi R, Caruthers SD, Janardhan V, et al. Intraventricular CSF pulsation artifact on fast fluid-attenuated inversion-recovery $M R$ images: analysis of 100 consecutive normal studies. AJNR Am J Neuroradiol 2000;21:503-08

28. Kallmes DF, Hui FK, Mugler JP 3rd. Suppression of cerebrospinal fluid and blood flow artifacts in FLAIR MR imaging with a singleslab three-dimensional pulse sequence: initial experience. Radiology 2001;221:251-55 